\title{
Hip fracture protection by alendronate treatment in postmenopausal women with osteoporosis: a review of the literature
}

\author{
Jun Iwamoto' \\ Yoshihiro Sato ${ }^{2}$ \\ Tsuyoshi Takeda' \\ Hideo Matsumoto \\ 'Department of Sports Medicine, Keio \\ University School of Medicine, Tokyo, \\ Japan; ${ }^{2}$ Department of Neurology, \\ Mitate Hospital, Fukuoka, Japan
}

\begin{abstract}
Osteoporosis most commonly affects postmenopausal women, placing them at a significant risk of fractures. In particular, hip fractures are an important cause of mortality and morbidity among postmenopausal women. Anti-resorptive therapies that produce greater decreases in bone turnover markers together with greater increases in bone mineral density (BMD) are associated with greater reductions in fracture risk, especially at sites primarily composed of cortical bone such as the hip. Thus, treatment with potent anti-resorptive drugs like alendronate is a strategy for preventing hip fractures in postmenopausal women with osteoporosis. The purpose of this paper is to discuss the efficacy of alendronate against hip fractures and the mechanism for this anti-fracture efficacy in postmenopausal women with osteoporosis. A meta-analysis of randomized controlled trials has shown that alendronate reduces the risk of hip fractures by $55 \%$ in postmenopausal women with osteoporosis. According to the analyses of the Fracture Intervention Trial, each 1 standard deviation reduction in a 1-year change in bone-specific alkaline phosphatase (BSAP) is associated with 39\% fewer hip fractures in alendronate-treated postmenopausal women, and those with at least 30\% reduction in BSAP have a $74 \%$ lower risk of hip fractures relative to those with less than $30 \%$. Alendronate is effective in reducing the risk of hip fractures across a spectrum of ages. The mechanism for this anti-fracture efficacy has been clarified; alendronate strongly suppresses bone turnover and subsequently increases hip BMD, decreases cortical porosity, improves parameters of hip structure geometry (cortical thickness, cross-sectional area, section modulus, and buckling ratio), and produces more uniform mineralization (increases the mean degree of mineralization of bone) in cortical bone. A once-weekly regimen of alendronate administration provides better patient compliance and persistence with the treatment than the once-daily dosing regimen, leading to greater efficacy against hip fractures. Thus, the efficacy of alendronate against hip fractures has been confirmed in postmenopausal women with osteoporosis, especially with a once-weekly dosing regimen.
\end{abstract}

Keywords: hip fracture, bone turnover, bone mineral density, cortical porosity, cortical thickness

\section{Introduction}

Osteoporosis most commonly affects postmenopausal women, placing them at a significant risk of fractures. In particular, hip fractures are an important cause of mortality and morbidity among postmenopausal women. Approximately $20 \%$ of patients with hip fractures die within a year, most of the deaths occurring within the first 6 months after a fracture (Cumming et al 1997). Among the survivors, 30\%-50\% never regain their prefracture functional status (Cooper et al 1993). Therefore, healthcare expenditure attributable to hip fractures is substantial.

Recent reports have shown that the rate of hip fractures is falling in Ontario and Finland (Jaglal et al 2005; Kannus et al 2006), probably because of recent indications that clinical attention to osteoporosis management is increasing and that specific programs of 
selective screening and aggressive treatment to prevent bone loss may really be able to reduce the fracture rate among the population most at risk (Melton et al 2005). However, there is no indication that the use of effective osteoporosis interventions is increasing dramatically in regions including Asia where the growth in the elderly population is most rapid.

Because elevated bone turnover markers and low bone mineral density (BMD) are independent predictors of hip fracture risk, and the risk is multiplied when both are present (Garnero et al 1996), bone turnover and BMD are important factors in decreasing the risk of hip fractures. Both reduction in bone turnover and increase in hip BMD may be necessary to decrease the fracture risk of the hip, which is primarily composed of cortical bone and may require greater proportionate changes than trabecular bone (Epstein 2007). In fact, drugs with a smaller effect on bone turnover reduce the risk of only vertebral fractures, whereas those with a greater effect reduce the risk of both vertebral and nonvertebral fractures including hip fractures (Delmas 2002). Furthermore, only those drugs associated with relatively large $\mathrm{BMD}$ increases together with greater reductions in bone turnover were effective for reducing the risk of nonvertebral fractures including hip fractures (Hochberg et al 2002).

Treatment with potent anti-resorptive drugs is a strategy for preventing hip fractures in postmenopausal women. Several therapeutic agents are currently available to treat or prevent postmenopausal osteoporosis (Iwamoto et al 2006, 2007). Among commercially available drugs, however, only alendronate, risedronate, intravenous zoledronate, strontium ranelate, and hormone replacement therapy (HRT) have shown anti-fracture efficacy against hip fractures in postmenopausal women (Black et al 1996, 2007; McClung et al 2001; Rossouw et al 2002; Reginster et al 2005). Alendronate and risedronate, which are the second- and third-generation bisphosphonates, respectively, have been used as first-line drugs in the treatment of osteoporosis for many years. In particular, evidence to explain the efficacy of alendronate against hip fractures has been accumulated for postmenopausal osteoporosis. Thus, this paper discusses, based on a review of the literature, the efficacy of alendronate against hip fractures and the mechanism for this anti-fracture efficacy in postmenopausal women with osteoporosis.

\section{Efficacy of alendronate against hip fractures in postmenopausal women with osteoporosis}

Treatment of osteoporosis is conducted in accordance with the principles of evidence-based medicine (EBM). EBM takes into account information derived from the highest- quality investigations with clinical judgment and patient values, to allow optimal clinical management. All available clinical evidence is classified hierarchically into various levels; strictly conducted systematic reviews with homogeneity represent the highest level, followed by strictly conducted randomized controlled trials (RCTs) with narrow confidence interval or meta-analyses with homogeneity of RCTs, which have long been considered as the "gold standard" in the context of clinical investigations (Centre for Evidence Based Medicine). The important points that must be considered in the choice of drugs for the treatment of osteoporosis are the effects of the drugs in reducing the incidence of fractures, the consistency of the results of RCTs of the drugs, and the long-term efficacy and safety of the drugs.

A meta-analysis conducted by Papapoulos et al (2005) demonstrated the efficacy of alendronate against hip fractures in postmenopausal women with osteoporosis. In total, 6,804 women with BMD T-score of -2.5 or less (age range: 39-91 years) from 6 RCTs were analyzed. The rates of hip fractures were 29 per 10,000 person-years at risk (PYR) in the alendronate group and 62 PYR in the control group. The overall risk reduction rate was $55 \%$ with the consistent results of RCTs (Figure 1). Reductions in risk of hip fractures with alendronate treatment were consistent across all studies. The efficacy of alendronate against hip fractures in

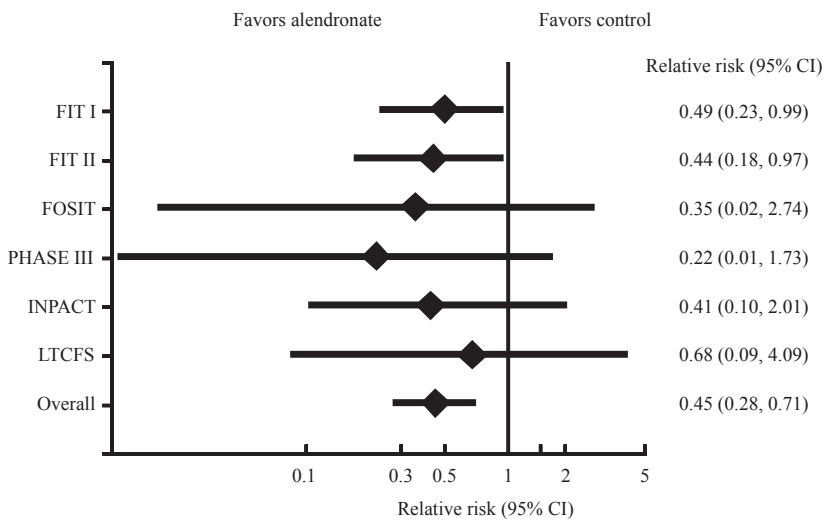

Figure I Meta-analysis of the efficacy of alendronate against hip fractures in postmenopausal women with osteoporosis. A meta-analysis conducted by Papapoulos et al (2005) demonstrated the efficacy of alendronate against hip fractures in postmenopausal women with osteoporosis who were naïve to bisphosphonate treatment. In total, 6,804 women with BMD T-score of -2.5 or less (age range: 39-9l years) from 6 multicenter RCTs were analyzed. The rates of hip fractures were 29 per 10,000 PYR in the alendronate group and 62 PYR in the control groups. The overall risk reduction rate was $55 \%$ (relative risk $0.45,95 \% \mathrm{Cl} 0.28-0.7 \mathrm{I}$ ) with consistent results for RCTs. Reductions in risk of hip fractures with alendronate treatment were consistent across all studies; the $P$ value of consistency is $0.98 \mathrm{I}$. Reproduced with permission from Papapoulos SE, Quandt SA, Liberman UA, et al. 2005. Meta-analysis of the efficacy of alendronate for the prevention of hip fractures in postmenopausal women. Osteoporos Int, 16:468-74. Copyright (c) 2005 Springer International.

Abbreviations: $\mathrm{BMD}$, bone mineral density; $\mathrm{Cl}$, confidence interval; $\mathrm{PYR}$, person-year at risk; RCT, randomized controlled trial. 
postmenopausal women with osteoporosis is supported by a report of the World Health Organization (WHO) Scientific Group (WHO Scientific Group 2003). RCTs have also demonstrated a similar incidence of gastrointestinal tract adverse events in patients treated with alendronate and a placebo, with no severe adverse events seen in the alendronate group (Liberman et al 1995; Black et al 1996; Cummings et al 1998). The long-term (10-year) safety of alendronate has been confirmed (Bone et al 2004; Black et al 2006); reductions in bone turnover remain stable throughout 10 years of alendronate treatment and are associated with continued gains in lumbar spine and hip BMD. Moreover, vertebral and nonvertebral fracture safety data suggest no loss of benefit.

Thus, alendronate could be a candidate for postmenopausal women with osteoporosis who are at a higher risk of hip fractures. Vitamin D and calcium supplementation would also be needed in patients with vitamin D insufficiency/deficiency and low calcium intake. According to the analyses of the Fracture Intervention Trial, postmenopausal women treated with alendronate with the greatest percentage reduction in bone-specific alkaline phosphatase (BSAP) have the lowest risk of hip fractures, while those with the smallest reduction in BSAP have the highest risk of hip fractures (Figure 2) (Bauer et al 2004). Each 1 standard

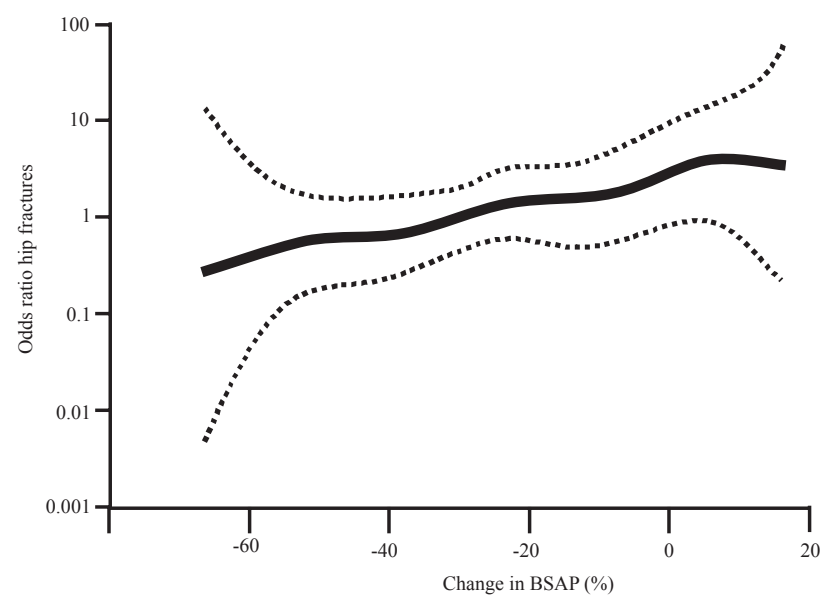

Figure 2 One-year change in BSAP and hip fracture risk among alendronate-treated postmenopausal women. The data from the FIT, a randomized, double-blind clinical trial of 6459 women recruited at II US clinical centers, were analyzed. The subjects were postmenopausal women (age range: $55-80$ years) with femoral neck BMD $=0.68$ $\mathrm{g} / \mathrm{cm}^{2}$ (Hologic QDR-2000) who were naïve to bisphosphonate treatment. Percent change in BSAP and predicted risk (log odds ratio) of hip fractures (solid line) and $95 \% \mathrm{Cl}$ (dotted lines) are reported from logistic regression model. Postmenopausal women treated with alendronate with the greatest percentage reduction in BSAP had the lowest risk of hip fractures, while those with the smallest reduction in BSAP had the highest risk of hip fractures. Reproduced from Bauer DC, Black DM, Garnero P et al. 2004. Change in bone turnover and hip, non-spine, and vertebral fracture in alendronate-treated women: the fracture intervention trial.J Bone Miner Res, 19:1250-8 with permission of the American Society for Bone and Mineral Research.

Abbreviations: BMD, bone mineral density; BSAP, bone-specific alkaline phosphatase $\mathrm{Cl}$, confidence interval; FIT, Fracture Intervention Trial.

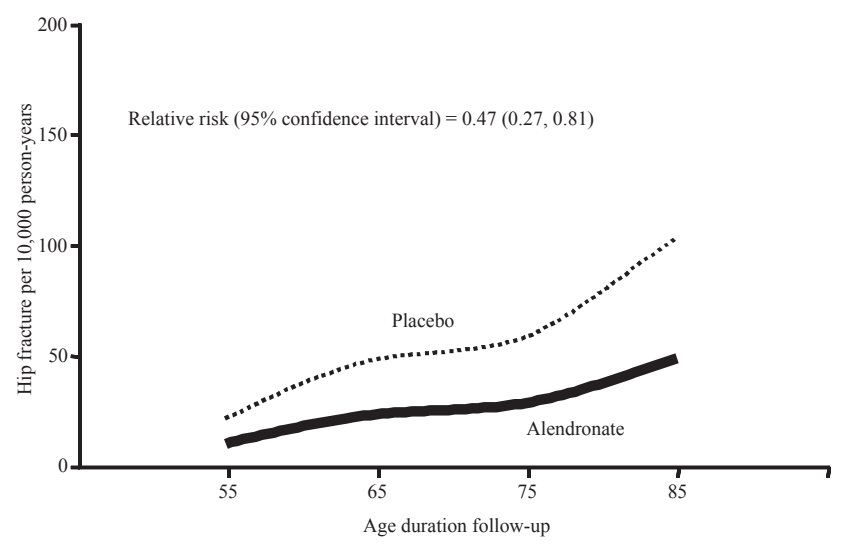

Figure 3 Rates of hip fractures per 10,000 person-years by attained age. A total of 18 randomized, placebo-controlled, double-blind, clinical trials of anti-resorptive agents that reported both changes in BMD (or BCM) and incidence of non-vertebral fractures were identified from a systematic literature review and from abstracts in conference proceedings (Hochberg et al 2005). These studies enrolled 26,494 women (age range: $3 \mathrm{I}-85$ years) who were naïve to anti-resorptive therapy and accumulated a total follow-up of 69,369 women-years, during which 2,415 women experienced one or more new nonvertebral fractures. Incidence curves were generated using a Cox proportional hazard model of the age-specific hip fracture data. The reductions in risk of hip fractures among alendronate-treated patients were significant relative to the placebo group for hip fractures; relative risk $(95 \% \mathrm{Cl})=0.47(0.27$, 0.8I). Reproduced from Hochberg MC, Thompson DE, Black DM, et al. 2005. Effect of alendronate on the age-specific incidence of symptomatic osteoporotic fractures. J Bone Miner Res, 20:97I-6 with permission of the American Society for Bone and Mineral Research.

Abbreviations: BCM, biochemical marker; $\mathrm{BMD}$, bone mineral density; $\mathrm{Cl}$, confidence interval.

deviation reduction in a 1-year change in BSAP is associated with 39\% fewer hip fractures in alendronate-treated postmenopausal women (Bauer et al 2004). Alendronatetreated postmenopausal women with at least $30 \%$ reduction in BSAP (56\% of alendronate-treated subjects) have a $74 \%$ lower risk of hip fractures relative to those with less than 30\% (Bauer et al 2004). Alendronate is effective in reducing the risk of hip fractures across a spectrum of ages (Figure 3 ) (Hochberg et al 2005). The effectiveness appears to be greater in patients with a femoral neck BMD $\mathrm{T}$ score $\leq-2.5$ than in patients with a femoral neck BMD $\mathrm{T}$ score $\leq-2.0$ (Hochberg et al 2005).

\section{Adherence of patients with alendronate treatment}

A retrospective analysis of a large population of new users of alendronate with diagnosed osteoporosis has confirmed that both compliance and persistence, in actual practice, is low and inadequate (Rabenda et al 2008). Siris et al (2006) analyzed the fracture probability across the full range of possible compliance values expressed as a medication possession ratio (MPR) (full MPR range: $0.0-1.0$ or $0 \%-100 \%$ ), which is defined as the number of days' supply of a medication with 
bisphosphonates during a certain period (Sclar et al 1991). At an MPR from $0 \%$ to $50 \%$, the probability of fracture during a period of 24 months remains consistent at about $11 \%$ and declines progressively once a threshold value of $50 \%$ is achieved (Siris et al 2006). Less than half the women were found to be compliant with bisphosphonate therapy (MPR $\geq 0.8$ ) and approximately $40 \%$ of women persisted with treatment for 12 months without a substantial gap in therapy (Rabenda et al 2008). Poor adherence to alendronate treatment is associated with an increased risk of hip fractures (Rabenda et al 2008). Thus, adherence (compliance and persistence) with treatment has represented a major challenge in patients receiving alendronate.

Once-weekly alendronate, which is the preferred dosing regimen, has been reported to be theoretically equivalent to daily dosing, providing postmenopausal osteoporotic women with a more convenient dosing option that enhances their adherence to treatment (Kendler et al 2004; Rossini et al 2006). Once-weekly alendronate has safety and tolerance profiles similar to daily alendronate in postmenopausal women with osteoporosis (Schnitzer et al 2000; Rizzoli et al 2002; Simon et al 2002).

It has been confirmed that compliance with bisphosphonate therapy is associated with fracture risk. Rabenda et al

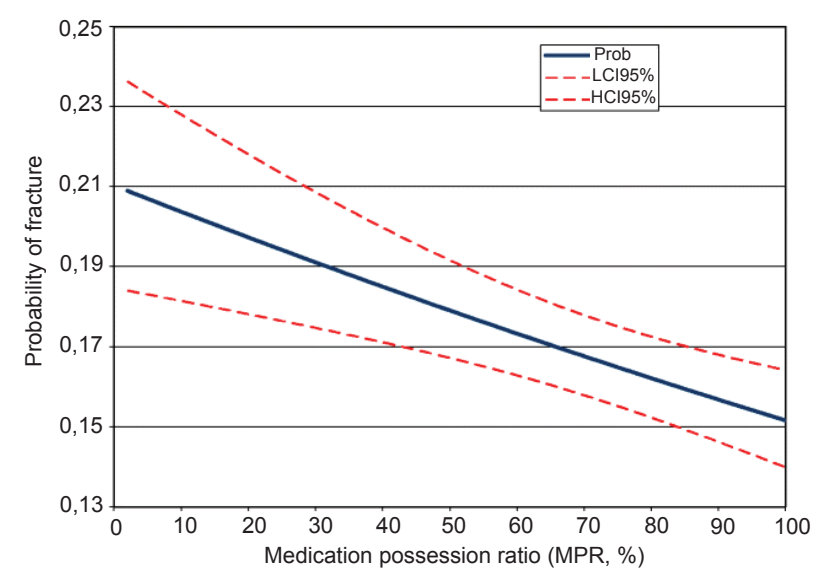

Figure 4 Probability of hip fractures according to medication possession ratio (MPR). A retrospective analysis of a large population of new users of alendronate with diagnosed osteoporosis was conducted to investigate adherence to bisphosphonate therapy and the impact of adherence on the risk of hip fractures (Rabenda et al 2008).An exhaustive search of the Belgian national social security database was conducted.A total of 29, 157 women (age range: 45 years or older) who were naïve to bisphosphonate treatment were included for the analysis of compliance (daily group $n=14,136$; weekly group $n=15,021$ ). The mean MPR at 12 months was $64.7 \%$. There was a negative linear estimated relationship between the probability of hip fractures and the value of the MPR. Reproduced with permission from Rabenda V, Mertens R, Fabri V, et al. 2008. Adherence to bisphosphonates therapy and hip fracture risk in osteoporotic women. Osteoporos Int, 19:81 I-18. Copyright (C) 2008 Springer International.

Abbreviations: $\mathrm{HCl}$, higher confidence interval (upper line); $\mathrm{LCl}$, lower confidence interval (lower line).
(2008) have reported that the MPR is a significant predictor of the occurrence of hip fractures in postmenopausal women treated with alendronate. The logistic regression model has estimated that for each decrease in the MPR of $1 \%$, the adjusted risk of hip fractures increases by $0.4 \%$. As shown in Figure 4 (Rabenda et al 2008), there is a negative estimated linear relationship between the probability of hip fractures and the value of the MPR. The treatment regimen is also an important determinant of hip fracture occurrence: postmenopausal women who receive weekly alendronate are $16.4 \%$ less likely to suffer from hip fractures than those on the daily regimen (Rabenda et al 2008).

Rabenda et al (2008) have also shown that persistence to alendronate treatment is a significant predictor of incurring hip fractures. The relative risk reduction for hip fractures is $60 \%$ for persistent patients compared with nonpersistent patients (hazard ratio: 0.404; 95\% CI: 0.357 , 0.457; $\mathrm{p}<0.0001)$.

Thus, both high compliance and persistence with prescribed osteoporosis medication are significantly associated with reduced hip fracture risk. The once-weekly alendronate regimen provides benefit in terms of better adherence (compliance and persistence) of the patients to the treatment regimen than the once-daily dosing regimen, leading to greater efficacy against hip fractures.

\section{Mechanism for the efficacy of alendronate against hip fractures}

Osteoporosis is defined as a skeletal disorder characterized by compromised bone strength, predisposing a person to an increased risk of fracture (NIH Consensus Development 2001). Properties related to bone strength include rate of bone turnover, BMD, geometry, microarchitecture, and mean degree of mineralization (Epstein 2005). Theoretically, microdamage might weaken bone, but no relationship to bone strength or fracture risk has been established (Epstein 2005).

The proximal femur of the hip is primarily composed of cortical bone. In cortical bone, high bone turnover, as observed after the menopause, is associated with decreased $\mathrm{BMD}$, increased cortical porosity due to increased bone remodeling in the Haversian canal, decreased cortical thickness due to increased endocortical bone remodeling, and decreased mean degree of mineralization (Hedlund et al 1989; Bousson et al 2000; Boivin et al 2000; Szulc et al 2006). Increased cortical thinning, increased porosity, and decreased mean degree of mineralization of bone (MDMB) are observed in the cortex in cases of hip fractures 
Table I Hypothesis proposed by Boivin et al about the potential mechanism by which anti-resorptive drugs act on bone remodeling and increase the mean degree of mineralization of bone. Reproduced with permission from Boivin GY, Chavassieux PM, Santora $A C$, et al. 2000.Alendronate increases bone strength by increasing the mean degree of mineralization of bone tissue in osteoporotic women. Bone, 27:687-94. Copyright (C) 2000 Elsevier.

$\begin{gathered}\text { Birthrate of } \\ \text { (Menopause) } \\ \text { the basic multicellular unit } \\ \text { Lifetime of } \\ \text { the basic structural unit } \\ \text { Duration of the secondary } \\ \text { mineralization }\end{gathered}$
Mean degree of
mineralization of bone

(Loveridge et al 2004; Bell et al 1999a; Bell et al 1999b; Bell 1999c), which might be associated with increased indices of Haversian remodeling. These changes caused by high bone remodeling in terms of high bone turnover translate to alterations in bone strength and risk of fractures.

Alendronate inhibits osteoclastic bone resorption, strongly reduces bone turnover, increases hip BMD, decreases cortical porosity, and produces more uniform mineralization (ie, increases the MDMB) in cortical bone, possibly contributing to a reduction in the risk of hip fractures (Liberman et al 1996; Black et al 1996; Cummings et al 1998; Boivin et al 2000; Roschger et al 2001). In alendronate-treated postmenopausal women, the distribution of the degree of mineralization in cortical bone shows a clear shift toward the highest mineralization values and a decrease in the number of bone structure units having low values of mineralization (Boivin et al

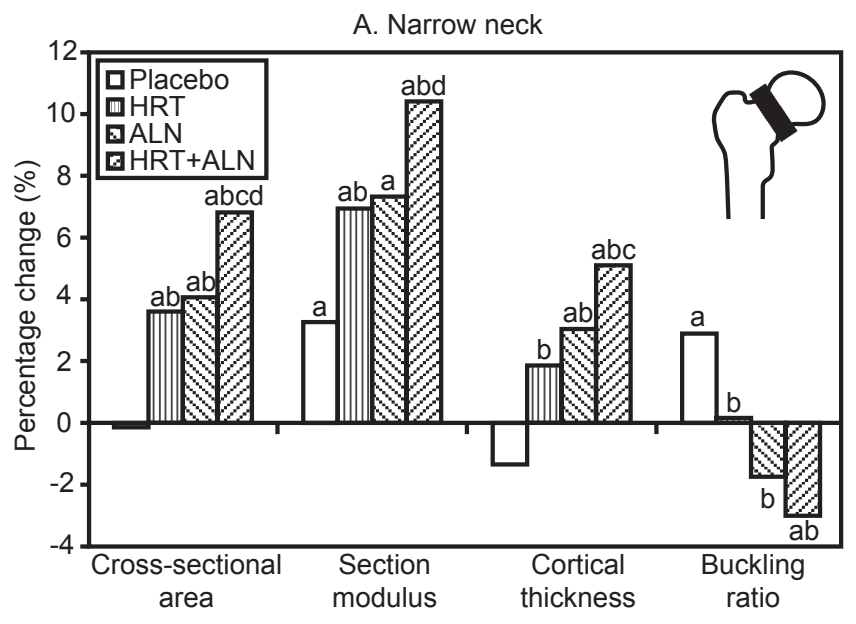

B. Intertrochanteric region

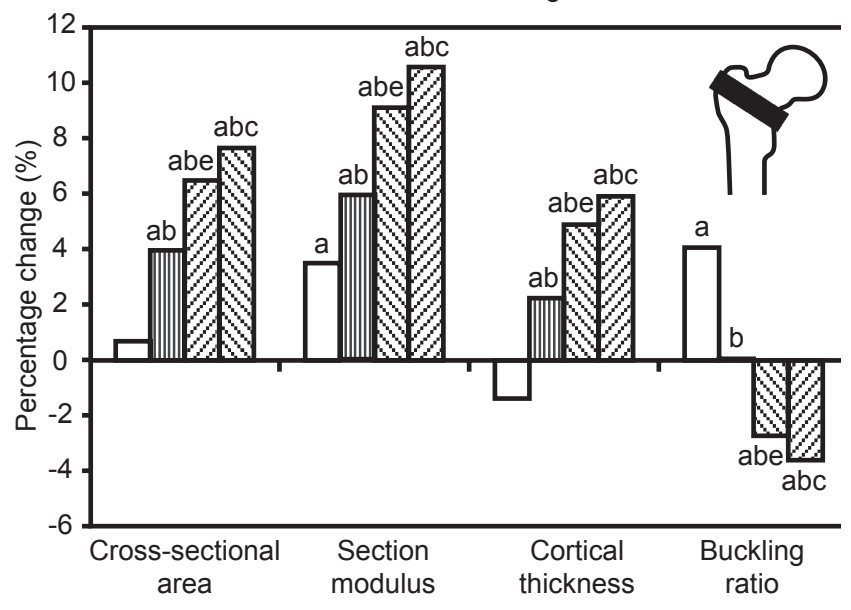

C. Femoral shaft

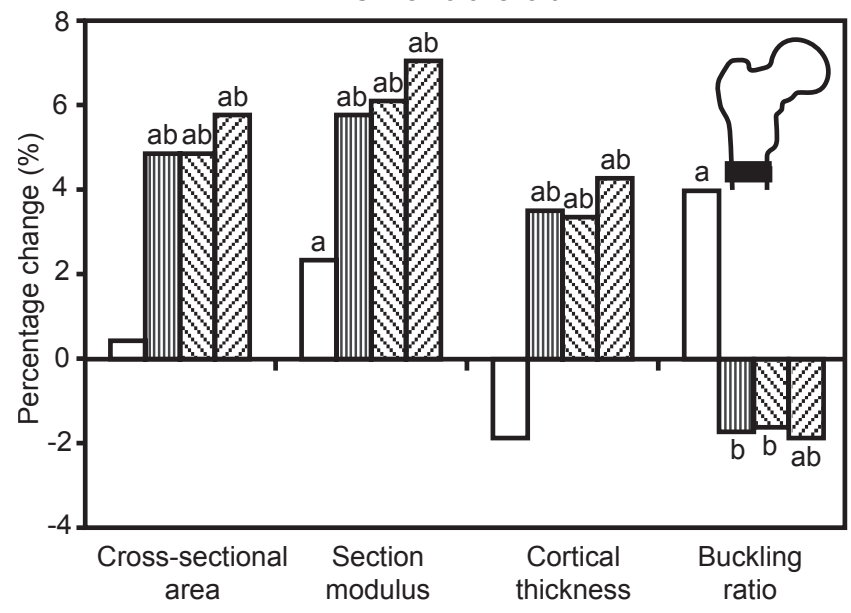

Figure 5 Percentage change in cross-sectional parameters by hip structure analysis. A double-blind, placebo-controlled, randomized clinical single-center trial was conducted over 3 years to examine the effect of HRT, ALN, or combination therapy on hip structure geometry in 373 community-dwelling women (age range: 65-90 years) who were naive to HRT or bisphosphonate treatment. To minimize discontinuation after randomization, all participants entered a 3-month, open-label run-in phase involving HRT, ALN placebo, and appropriate calcium and vitamin $D$ supplementation. Cross-sectional parameters were measured by HSA. a: $\mathrm{p}<0.05$ from baseline, b: $\mathrm{p}<0.05$ vs placebo, c: $\mathrm{p}<0.05 \mathrm{HRT}+\mathrm{ALN}$ vs HRT, d: $\mathrm{p}<0.05 \mathrm{HRT}+\mathrm{ALN}$ vs ALN, e: $\mathrm{p}<0.05 \mathrm{ALN}$ vs HRT. Alendronate improved parameters of hip structure geometry as evaluated by the HSA such as cortical thickness, cross-sectional area, section modulus, and buckling ratio in the narrow neck (A), intertrochanteric region (B), and femoral shaft (C). In addition, improvements of the above parameters in the narrow neck and intertrochanteric region were greater with combination therapy with HRT. Reproduced from Greenspan SL, Beck TJ, Resnick $\mathrm{NM}$, et al. 2005. Effect of hormone replacement, alendronate, or combination therapy on hip structural geometry: a 3-year, double-blind, placebo-controlled clinical trial. J Bone Miner Res, 20:1525-32 with permission of the American Society for Bone and Mineral Research.

Abbreviations: ALN, alendronate; HSA, hip structure analysis; HRT, hormone replacement therapy.

2000). The MDMB augmentation probably accounts for most of the increase in BMD seen with alendronate (Boivin et al 2000). According to the hypothesis proposed by Boivin et al (2000), the reduction in the activation frequency caused by the anti-resorptive effect of alendronate is followed by a prolonged secondary mineralization that increases the percentage of bone 
structure units having reached a maximum degree of secondary mineralization and, though this mechanism, augmentation of the MDMB (Table 1).

Recently, it has been possible to extract cross-sectional geometry from dual-energy X-ray absorptiometry (DXA) scan imaging. Hip structure analysis (HAS) is a computer program designed to perform this function with DXA scans of the hip. Greenspan et al (2005) have demonstrated that alendronate improves parameters of hip structure geometry as evaluated by the HAS, such as cortical thickness, cross-sectional area, section modulus, and buckling ratio in the narrow neck, intertrochanteric region, and femoral shaft (Figure 5) (Greenspan et al 2005). These data provide additional information on a potential mechanism for hip fracture reduction with alendronate. In addition, improvements in the above parameters in the narrow neck and intertrochanteric region are greater in combination therapy with HRT (Figure 5) (Greenspan et al 2005). This result supports the concept that anti-resorptive therapies that produce larger decreases in bone turnover markers together with larger increases in BMD are associated with greater reductions in hip fracture risk, especially at sites primarily composed of cortical bone (Hochberg et al 2002).

Osteocyte viability has been observed to be an indicator of bone strength, with viability as the result of maintaining physiological levels of loading and osteocyte apoptosis as the result of a decrease in loading (Epstein 2007). Osteocyte apoptosis and decrease are major factors in the bone loss and fractures associated with aging (Epstein 2007). A recent animal study demonstrated that low doses of risedronate or alendronate suppressed osteocyte apoptosis induced by fatigue loading of the ulna in rats (Follet 2007). However, the effect of alendronate on osteocyte apoptosis remains to be established in clinical studies.

\section{Conclusions}

Evidence derived from the literature, stratified and based on strict EBM guidelines, suggests the efficacy of alendronate against hip fractures in postmenopausal women with osteoporosis with an overall risk reduction rate of $55 \%$. According to the analyses of the Fracture Intervention Trial, each 1 standard deviation reduction in a 1-year change in BSAP is associated with 39\% fewer hip fractures in alendronate-treated postmenopausal women, and those with at least a $30 \%$ reduction in BSAP have a $74 \%$ lower risk of hip fractures relative to those with less than $30 \%$. Alendronate is effective in reducing the risk of hip fractures across a spectrum of ages. The mechanism for this anti-fracture efficacy has been clarified; alendronate suppresses bone turnover and subsequently increases hip BMD, decreases cortical porosity, improves parameters of hip structure geometry (cortical thickness, cross-sectional area, section modulus, and buckling ratio), and produces more uniform mineralization (increases the MDMB) in cortical bone. A once-weekly alendronate regimen provides better adherence (compliance and persistence) of the patients to the treatment than the once-daily dosing regimen, leading to greater efficacy against hip fractures. Thus, the efficacy of alendronate against hip fractures has been confirmed in postmenopausal women with osteoporosis, especially with once-weekly dosing regimen.

\section{Abbreviations}

BMD, bone mineral density; BSAP, bone-specific alkaline phosphatase; HRT, hormone replacement therapy; EBM: evidence-based medicine; RCT, randomized controlled trial; PYR, person-years at risk; WHO, World Health Organization; MPR, medication possession ratio; MDMB, mean degree mineralization of bone; DXA, dual-energy X-ray absorptiometry; HSA, hip structure analysis.

\section{Disclosures}

The authors sought no assistance in the preparation of this manuscript. The study was not supported by any grant. None of the authors has any conflict of interests in relation to the publication of this paper.

\section{References}

Bauer DC, Black DM, Garnero P, et al. 2004. Change in bone turnover and hip, non-spine, and vertebral fracture in alendronate-treated women: the fracture intervention trial. J Bone Miner Res, 19:1250-8.

Bell KL, Loveridge N, Power J, et al. 1999a. Intracapsular hip fracture: increased cortical remodeling in the thinned and porous anterior region of the femoral neck. Osteoporos Int, 10:248-57.

Bell KL, Loveridge N, Power J, et al. 1999b. Regional differences in cortical porosity in the fractured femoral neck. Bone, 24:57-64.

Bell KL, Loveridge N, Power J, et al. 1999c. Structure of the femoral neck in hip fracture: cortical bone loss in the inferoanterior to superoposterior axis. J Bone Miner Res, 14:1111-9.

Black DM, Cummings SR, Karpf DB, et al. 1996. Randomised trial of effect of alendronate on risk of fracture in women with existing vertebral fractures. Lancet, 348:1535-41.

Black DM, Delmas PD, Eastell R, et al. 2007. Once-yearly zoledronic acid for treatment of postmenopausal osteoporosis. $N$ Engl J Med, 356:1809-22.

Black DM, Schwartz AV, Ensrud KE, et al. 2006. Effects of continuing or stopping alendronate after 5 years of treatment: the Fracture Intervention Trial Long-term Extension (FLEX): a randomized trial. JAMA, 296:2927-38

Boivin GY, Chavassieux PM, Santora AC, et al. 2000. Alendronate increases bone strength by increasing the mean degree of mineralization of bone tissue in osteoporotic women. Bone, 27:687-94.

Bone HG, Hosking D, Devogelaer JP, et al. 2004. Ten years' experience with alendronate for osteoporosis in postmenopausal women. $N \mathrm{Engl}$ J Med, 350:1189-99.

Bousson V, Bergot C, Meunier A, et al. 2000. CT of the middiaphyseal femur: cortical bone mineral density and relation to porosity. Radiology, 217:179-87. 
Centre for Evidence-Based Medicine. Oxford-Centre Evidence Based Medicine. Levels of Evidence and Grades of Recommendation. http://www. cebm.net/levels_of_evidence.asp.

Cooper C, Atkinson EJ, Jacobsen SJ, et al. 1993. Population-based study of survival after osteoporotic fractures. Am J Epidemiol, 137:1001-5.

Cumming RG, Nevitt MC, Cummings SR. 1997. Epidemiology of hip fractures. Epidemiol Rev, 19:244-57.

Cummings SR, Black DM, Thompson DE, et al. 1998. Effect of alendronate on risk of fracture in women with low bone density but without vertebral fractures: results from the Fracture Intervention Trial. JAMA, 280:2077-82.

Delmas PD. 2002. Different effects of antiresorptive therapies on vertebral and nonvertebral fractures in postmenopausal osteoporosis. Bone, 30:14-17.

Epstein S. 2005. The roles of bone mineral density, bone turnover, and other properties in reducing fracture risk during antiresorptive therapy. Mayo Clin Proc, 80:379-88.

Epstein S. 2007. Is cortical bone hip? What determines cortical bone properties? Bone, 41(Suppl 1): S3-8.

Follet H, Li J, Phipps RJ, et al. 2007. Risedronate and alendronate suppress osteocyte apoptosis following cyclic fatigue loading. Bone, 40:1172-7.

Garnero P, Hausherr E, Chapuy MC, et al. 1996. Markers of bone resorption predict hip fracture in elderly women: the EPIDOS Prospective Study. $J$ Bone Miner Res, 11:1531-8.

Greenspan SL, Beck TJ, Resnick NM, et al. 2005. Effect of hormone replacement, alendronate, or combination therapy on hip structural geometry: a 3-year, double-blind, placebo-controlled clinical trial J Bone Miner Res, 20:1525-32.

Hedlund LR, Gallagher JC. 1989. The effect of age and menopause on bone mineral density of the proximal femur. J Bone Miner Res, 4:639-42.

Hochberg MC, Greenspan S, Wasnich RD, et al. 2002. Changes in bone density and turnover explain the reductions in incidence of nonvertebral fractures that occur during treatment with antiresorptive agents. J Clin Endocrinol Metab, 87:1586-92.

Hochberg MC, Thompson DE, Black DM, et al. 2005. Effect of alendronate on the age-specific incidence of symptomatic osteoporotic fractures. $J$ Bone Miner Res, 20:971-6.

Iwamoto J, Takeda T, Sato Y. 2006. Efficacy and safety of alendronate and risedronate for postmenopausal osteoporosis. Curr Med Res Opin, 22:919-28.

Iwamoto J, Takeda T, Sato Y.2007. Effects of antifracture drugs in postmenopausal, male and glucocorticoid-induced osteoporosis - Usefulness of alendronate and risedronate. Expert Opin Pharmacother, 8:2743-56.

Jaglal SB, Weller I, Mamdani M, et al. 2005. Population trends in BMD testing, treatment, and hip and wrist fracture rates: are the hip fracture projections wrong? J Bone Miner Res, 20:898-905.

Kannus P, Niemi S, Parkkari J, et al. 2006. Nationwide decline in incidence of hip fracture. J Bone Miner Res, 21:1836-8.

Kendler D, Kung AW, Fuleihan Gel-H, et al. 2004. Patients with osteoporosis prefer once weekly to once daily dosing with alendronate. Maturitas, 48:243-51.

Liberman UA, Weiss SR, Broll J, et al. 1995. Effect of oral alendronate on bone mineral density and the incidence of fractures in postmenopausal osteoporosis. N Engl J Med, 333:1437-43.
Loveridge N, Power J, Reeve J, et al. 2004. Bone mineralization density and femoral neck fragility. Bone, 35:929-41.

McClung MR, Geusens P, Miller PD, et al. 2001. Effect of risedronate on the risk of hip fracture in elderly women. Hip Intervention Program Study Group. N Engl J Med, 344:333-40.

Melton LJ 3rd, Kanis JA, Johnell O. 2005. Potential impact of osteoporosis treatment on hip fracture trends. J Bone Miner Res, 20:895-7.

NIH Consensus Development. 2001. Panel on Osteoporosis Prevention, Diagnosis, and Therapy. Osteoporosis prevention, diagnosis, and therapy. JAMA, 285:785-95.

Papapoulos SE, Quandt SA, Liberman UA, et al. 2005. Meta-analysis of the efficacy of alendronate for the prevention of hip fractures in postmenopausal women. Osteoporos Int, 16:468-74.

Rabenda V, Mertens R, Fabri V, et al. 2008. Adherence to bisphosphonates therapy and hip fracture risk in osteoporotic women. Osteoporos Int, 19:811-18.

Rizzoli R, Greenspan SL, Bone G III, et al. 2002. Two-year results of once-weekly administration of alendronate $70 \mathrm{mg}$ for the treatment of postmenopausal osteoporosis. J Bone Miner Res, 17:1988-96.

Reginster JY, Seeman E, De Vernejoul MC, et al. 2005. Strontium ranelate reduces the risk of nonvertebral fractures in postmenopausal women with osteoporosis: Treatment of Peripheral Osteoporosis (TROPOS) study. J Clin Endocrinol Metab, 90:2816-22.

Roschger P, Rinnerthaler S, Yates J, et al. 2001. Alendronate increases degree and uniformity of mineralization in cancellous bone and decreases the porosity in cortical bone of osteoporotic women. Bone, 29:185-91.

Rossini M, Bianchi G, Di Munno O, et al. 2006. Determinants of adherence to osteoporosis treatment in clinical practice. Osteoporos Int, 17:914-21.

Rossouw JE, Anderson GL, Prentice RL, et al. 2002. Risks and benefits of estrogen plus progestin in healthy postmenopausal women: principal results From the Women's Health Initiative randomized controlled trial. JAMA, 288:321-33.

Schnitzer T, Bone HG, Crepaldi G, et al. 2000. Therapeutic equivalence of alendronate $70 \mathrm{mg}$ once-weekly and alendronate $10 \mathrm{mg}$ daily in the treatment of osteoporosis. Aging, 12:1-12.

Sclar DA, Chin A, Skaer TL, et al. 1991. Effect of health education in promoting prescription refill compliance among patients with hypertension. Clin Ther, 13:489-95.

Simon JA, Lewiecki EM, Smith ME, et al. 2002. Patient preference for once-weekly alendronate $70 \mathrm{mg}$ versus once-daily alendronate $10 \mathrm{mg}$ : A multicenter, randomized, open-label, crossover study. Clin Ther, 24:1871-86.

Siris ES, Harris ST, Rosen CJ, et al. 2006. Adherence to bisphosphonate therapy and fracture rates in osteoporotic women: relationship to vertebral and nonvertebral fractures from 2 US claims databases. Mayo Clin Proc, 81:1013-22.

Szulc P, Seeman E, Duboeuf F, et al. 2006. Bone fragility: failure of periosteal apposition to compensate for increased endocortical resorption in postmenopausal women. J Bone Miner Res, 21:1856-63.

WHO Scientific Group. 2003. Prevention and management of osteoporosis. World Health Organ Tech Rep Ser, 921:86-109. 
